development process, when applied, will significantly advance pediatric preparedness. Ultimately, these pediatric specific measures must exist and be used to assess current levels of performance and guide resource allocation and targeted improvement efforts.

Prehosp Disaster Med 2011;26(Suppl. 1):s35-s36

doi:10.1017/S1049023X11000884

(A127) Major Trauma in a Swedish Paediatric PopulationA Survey of Children Admitted to a Neuro Intensive Care Unit (NICU)

\section{L.E. Franzén}

Paediatric Surgery, Göteborg, Sweden

Purpose: To describe the demographics, mechanism, pattern, and severity of injury, prehospital and hospital care (first 24 hours) and the patient outcome in severely injured children in a NICU. This study was made to complete the study of Swedish children admitted to a paediatric intensive care unit (PICU) due to major trauma in the same region and during the same period. Method: The medical records of 124 traumatized children (0-16 years of age), admitted to the NICU in Gothenburg 1992-2001, were retrospectively examined. The Injury Severity Score (ISS), Glasgow Paediatric Coma Scale (GSC), Revised Trauma Score (T-RTS/RTS), Paediatric Trauma Score (PTS), Trauma Score Injury Severity Score (TRISS) and Paediatric Risk of Mortality Score (PRISM) estimated the severity of injury.

Results: About 7/100 000 children with severe injuries were admitted to the NICU each year from 1992-2001 inclusive. Epidemiology showed a similar pattern as in other OECD countries. Severity of injury was recorded as an ISS median of 17. Mortality rate in our series was $6 \%$.

Conclusion: Major trauma with admission to a NICU is rare in Swedish children. With management in conjunction with a pediatric centre, these children have a good survival rate. Prehosp Disaster Med 2011;26(Suppl. 1):s36 doi:10.1017/S1049023X11001282

\section{(A128) Awareness and Preparedness of Western} Children's Hospitals for Disasters

\section{A. Fette}

Medical School, Weissach Im Tal, Germany

Disasters involving children are becoming more and more frequent. Thus, optimal preparedness will be a challenge for every Western pediatric disaster specialist. However, for any appropriate decision to be made, there must be a practical tool for accurately evaluating the levels of specific disaster awareness and preparedness. This tool is based on the idea that child injury prevention campaigns $[n=6]$ are usable as a platform for the simulation of specific pediatric disaster scenarios, and that different simulations might be able to modulate overall awareness and overall preparedness levels, as well as affect the training provided. Data are gathered from a disaster phase-related (Haddon-Matrix) set of questionnaires answered by key disaster response personnel [ $n=58]$. Overall awareness for a pediatric disaster scored highest for the "in the world" scenarios, with less, but with similar scores for "in the country" and "in the region" scenarios. Overall preparedness scored low for "in the world", with higher scores for "in the country" and "in the region". Both, overall awareness and overall preparedness scored inconsistently for "in the hospital" in the first instance, but later in the matrix, "in the hospital" had the highest scores. In general, basic knowledge about disaster plans is moderate, and knowledge about existence and activation of preparedness measures is above average. Individual position-taking and feelings of personal competency in position-taking is low, especially among junior staff. Currently, only a group of seniors are able report participation in a specific training. This platform is an upgradable tool for the awareness of and preparedness for pediatric disaster assessments, regarding phases, locations, and training, with promising trends for their modulation, especially among junior staff.

Prehosp Disaster Med 2011;26(Suppl. 1):s36

doi:10.1017/S1049023X11001294

(A129) A Pediatric Surgeon's Viewpoint of a Concealed Disaster

A. Fette, ${ }^{1}$ K. Paya ${ }^{2}$

1. Medical School, Weissach Im Tal, Germany

2. Astana, Kazakhstan

Childhood is one of the most vulnerable parts in a human's life. Thus, any physical and psychological harm against children requires special attention, especially if inflicted and not accidental. Such children should be considered multi-trauma victims and managed by a multidisciplinary team and trauma algorithm. In this team of specialized carers, the pediatric surgeon will import his/her expertise on general management and treatment and simultaneously refer basic knowledge to more junior doctors that might be in charge in the future. Fifty-eight injured victims (mean age $=1.5$ years of age, range $=1$ day-18 years of age, male:female ratio $=1: 1$ ) were analyzed in this study. Their injuries were subcategorized into battery (13), assault (11), neglect (3), sexual abuse (2), prevention failure (6), career-related (19), and miscellaneous (5). All victims were first seen by a pediatric surgeon before receiving multidisciplinary consultations. Treatment results and modalities varied according to the complexity of the diagnoses requiring a well-trained and skilled pediatric surgeon. Accompanying post-traumatic stress disorders within the children as well as psychological distress among the parents and grandparents were quite frequent. Besides medico-surgical treatment, empathic care is essential. In the majority of cases the children, benefited from pediatric surgical care.

Prehosp Disaster Med 2011;26(Suppl. 1):s36

doi:10.1017/S1049023X11001300

(A130) Advantages of Apparatus of External Fixation in Severe Injuries of Extremities in Children

A. Karabenyuk, A. Levitskiy

Paediatric Surgery, Kiev, Ukraine

Purpose: To study advantages of external fixation in severe injuries of extremities in children.

Material and Method: 305 children at the age from 3 to 17 years with polytrauma (ISS > 18) were studied. From them skeletal injuries took place in 198 patients, cranioskeletal trauma - 125, 